\title{
Optical demodulation system for digitally encoded suspension array in fluoroimmunoassay
}

Qinghua He

Dongmei Li

Yonghong $\mathrm{He}$

Tian Guan

Yilong Zhang

Zhiyuan Shen

Xuejing Chen

Siyu Liu

Bangrong Lu

Yanhong Ji 


\title{
Optical demodulation system for digitally encoded suspension array in fluoroimmunoassay
}

\author{
Qinghua He, ${ }^{a, b}$ Dongmei Li, ${ }^{a, b}$ Yonghong He, ${ }^{a, b, *}$ Tian Guan, ${ }^{b}$ Yilong Zhang, ${ }^{a, b}$ Zhiyuan Shen, ${ }^{a, b}$ \\ Xuejing Chen, ${ }^{a, b}$ Siyu Liu, ${ }^{b}$ Bangrong Lu, ${ }^{c}$ and Yanhong $\mathrm{Ji}^{\mathrm{c}, *}$ \\ ${ }^{a}$ Tsinghua University, Department of Physics, Beijing, China \\ ${ }^{\mathrm{b}}$ Tsinghua University, Shenzhen Key Laboratory for Minimal Invasive Medical Technologies, Institute of Optical imaging and Sensing, \\ Graduate School at Shenzhen, Shenzhen, China \\ 'South China Normal University, School of Physics and Telecommunication Engineering, Guangzhou, China
}

\begin{abstract}
A laser-induced breakdown spectroscopy and fluorescence spectroscopy-coupled optical system is reported to demodulate digitally encoded suspension array in fluoroimmunoassay. It takes advantage of the plasma emissions of assembled elemental materials to digitally decode the suspension array, providing a more stable and accurate recognition to target biomolecules. By separating the decoding procedure of suspension array and adsorption quantity calculation of biomolecules into two independent channels, the cross talk between decoding and label signals in traditional methods had been successfully avoided, which promoted the accuracy of both processes and realized more sensitive quantitative detection of target biomolecules. We carried a multiplexed detection of several types of anti-lgG to verify the quantitative analysis performance of the system. A limit of detection of $1.48 \times 10^{-10} \mathrm{M}$ was achieved, demonstrating the detection sensitivity of the optical demodulation system. ๑ The Authors. Published by SPIE under a Creative Commons Attribution 3.0 Unported License. Distribution or reproduction of this work in whole or in part requires full attribution of the original publication, including its DOI. [DOI: 10.1117/1.JBO.22.9.097003]
\end{abstract}

Keywords: optical demodulation; suspension array; laser-induced breakdown spectroscopy.

Paper 170283R received May 4, 2017; accepted for publication Sep. 5, 2017; published online Sep. 21, 2017.

\section{Introduction}

Benefiting from high sensitivity, low sample consumption, and high-throughput detection performance in biomolecular analysis, suspension array is gradually becoming a popular detection method in life science. ${ }^{1-5}$ The whole analysis system of suspension array is built on monodisperse microbeads, with diameters in tens of nanometers to hundreds of micrometers. ${ }^{6}$ After modification and bioprobe grafting on the surface, microspheres obtain the ability to specifically capture target biomolecules in samples. ${ }^{7}$ By measuring the amount of captured biomolecules, the concentration of target biomolecules in samples could be deduced proportionally. In fluoroimmunoassay analysis, the amounts of captured molecules are calculated by collecting the fluorescence emission from the label, ${ }^{8,9}$ which indicates that the quantitative analysis of analyte can be achieved by the fluorescence measurement of a single microbead. ${ }^{10,11}$ Once various types of microbeads can specifically capture different analytes in the same sample and can be recognized with each other, the microbead-based detection mode can endow suspension array the potential of multiplexing. ${ }^{12}$ To achieve the recognition of microbeads, a large amount of encoding and decoding methods had been proved by researchers. Currently, the most widely applicable encoding method is the fluorecent encoding method, which labeled suspension array with particular fluorescence signals. ${ }^{13-15}$ Hence, after sample detection, the recognition of suspension array and the counting of label fluorescence intensity should be carried together in fluorescence measurements. However, according to the theory of

\footnotetext{
*Address all correspondence to: Yonghong He, E-mail: heyh @sz.tsinghua.edu
} .cn; Yanhong Ji, E-mail: jiyh@scnu.edu.cn fluorescence resonance energy transfer, once there are overlaps between the absorption spectrum of fluorescent acceptor and emission spectrum of fluorescent donor, the fluorescent energy might transfer from donor to acceptor at a certain distance. This phenomenon always causes enhancements of long-wavelength fluorescence and quenching of short-wavelength fluorescence. ${ }^{16-18}$ Thus, the utilization of fluorescence encoding might produce cross talk with the label fluorescence, causing interference in both qualitative and quantitative analyses.

For the purpose of avoiding the cross talk, we designed a kind of digital encoded suspension array to segregate the decoding and label signals into two independent optical channels: the laser-induced breakdown spectroscopy (LIBS) channel and fluorescence channel. As it is well known, LIBS is an element analysis technique. By collecting the laser-induced plasma emissions, especially atomic lines, the existence of specific elements in materials could be detected with characteristic peaks and spectrum. ${ }^{19-21}$ Benefiting from the stability and linear shape of atomic lines, LIBS can provide accurate and stable information about contained elements in materials, being widely applied in environmental monitoring, ${ }^{22,23}$ geological prospecting,${ }^{24}$ and industry detection. ${ }^{25,26}$

In this study, we designed and built an optical demodulation system for digitally encoded suspension array in fluoroimmunoassay. A confocal LIBS system was combined with a fluorescence system to achieve the independent detection of decoding and label signal, accomplishing the qualitative and quantitative analysis of samples in separated channels. By assembling specific elementary materials into microbead-carriers and grafting bioprobes on the surface, we prepared different types of suspension array and carried multiplexed detection of fluorescencelabeled target biomolecules with them. The reacted suspension 
array was placed on glass slides and respectively scanned with a fluorescence stimulation laser and nanosecond pulse laser, which were set in a common light path. The independent stimulations and collections of fluorescence and laser-induced breakdown spectrum avoided the cross talk and provided a clean background for both decoding and counting of biomolecules. In experiments, various types of suspension arrays worked in the same sample to specifically recognize different types of anti-IgGs. The reacted suspension arrays were analyzed together on a glass slide by the optical demodulation system to demonstrate the performance of multiplexed analysis. In addition, the concentration response curve to anti-IgG detection was measured to verify the availability of the system in practical application.

\section{Experiment}

\subsection{Dual-Channel Design of the Optical Demodulation System}

The dual-channel (fluorescence and LIBS)-based demodulation system for suspension array in biomolecules detection was first proposed in this paper. The label fluorescence was collected in the fluorescence channel to count the captured biomolecules, accomplishing the quantitative analysis of analytes. In the LIBS channel, a nanosecond pulse laser stimulated assembled materials and produced plasma on the suspension array. By collecting the plasma emissions and transferring the laserinduced breakdown spectrum, the suspension array was digitally decoded, achieving the qualitative analysis of analyte. As shown in Fig. 1, the quantum dots label on the biomolecules was stimulated by a $405-\mathrm{nm}$ laser and the intensity was counted in the fluorescence channel. The higher intensity represents more captured biomolecules, which indicates higher concentration of analytes in the sample. The microbeads were stimulated by a 1064-nm pulse laser and the laser-induced breakdown spectrum was collected and analyzed in the LIBS channel. All types of spectra were corresponding to specific types of suspension arrays, and further revealed the types of absorbed biomolecules on the microbeads.

\subsection{Optical Setups of the Optical Demodulation System}

The optical setup is shown in Fig. 2. In the fluorescence channel of the system, the fluorescence label was stimulated and the emissions were collected to count the intensity, obtaining the concentration information of target biomolecules. The LIBS channel was set up for the collection and analysis of plasma emission

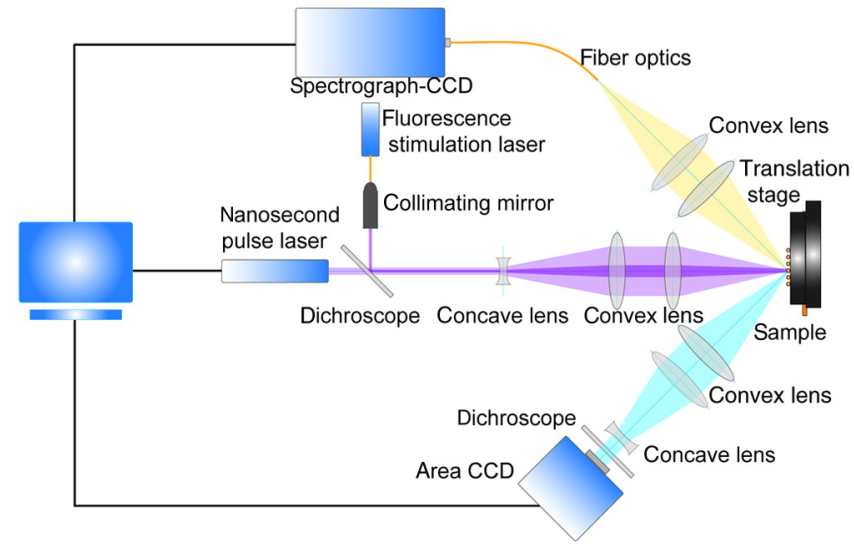

Fig. 2 The schematic of optical demodulation system for digitally encoded suspension array.

produced by encoded microbeads. The stimulation parts of two channels were coupled into a common optical path except the excitation source. A $1064 \mathrm{~nm}$ nanosecond pulse laser (Beamtech, China) with pulse width of $8 \mathrm{~ns}$ and tunable output repetition rate from 1 to $10 \mathrm{~Hz}$ was used to act as the stimulation light to induce plasma on the microbeads. With an output power of $100 \mathrm{~mJ}$ of every pulse, the pulse laser beam was outputted and expanded by a combination of concave lens and a convex lens to form a beam with a diameter of $40 \mathrm{~mm}$, and then was focused on the sample by a convex lens $(f=150 \mathrm{~mm})$. The spot diameter of the focused 1064-nm laser was $8 \mu \mathrm{m}$. A 405-nm fiber output laser (Xilong, China) beam with $110 \mathrm{~mW}$ output power was collimated to form a parallel beam with a diameter of $1 \mathrm{~mm}$ and reflected into the stimulation path by a dichroscope (high pass, cut-off wavelength $=500 \mathrm{~nm}, T_{\min }>85 \%, T_{\text {avg }}>90 \%$, $R_{\text {abs }}>90 \%$, and $R_{\text {avg }}>95 \%$ ). The spot diameter of the focused 405-nm laser was $30 \mu \mathrm{m}$. A convex lens $(f=150 \mathrm{~mm})$ with a diameter of $50.8 \mathrm{~mm}$ was used to collect the fluorescence emissions as much as possible. The emission was selected with another dichroscope (high pass, cut-off wavelength $=500 \mathrm{~nm}$, $T_{\min }>85 \%, T_{\text {avg }}>90 \%, R_{\text {abs }}>90 \%$, and $\left.R_{\text {avg }}>95 \%\right)$ to eliminate the reflected $405-\mathrm{nm}$ laser and coupled into area charge-coupled device (CCD) (SBIG, America) to count the intensity. The integral time of area CCD was 0.09 s. Equally, the plasma emission was collected by a convex lens with a diameter of $50.8 \mathrm{~mm}$ and coupled into multimode fiber optics (diameter $=62.8 \mu \mathrm{m}$ ). A spectrograph-CCD system (Tekspray, China) was used to analyze the emission and the integral time of the CCD was $55 \mu \mathrm{s}$. The sample was placed on the glass slide, which is coupled on the translation stage and the operation time sequence is listed as follows: the sample moved under the
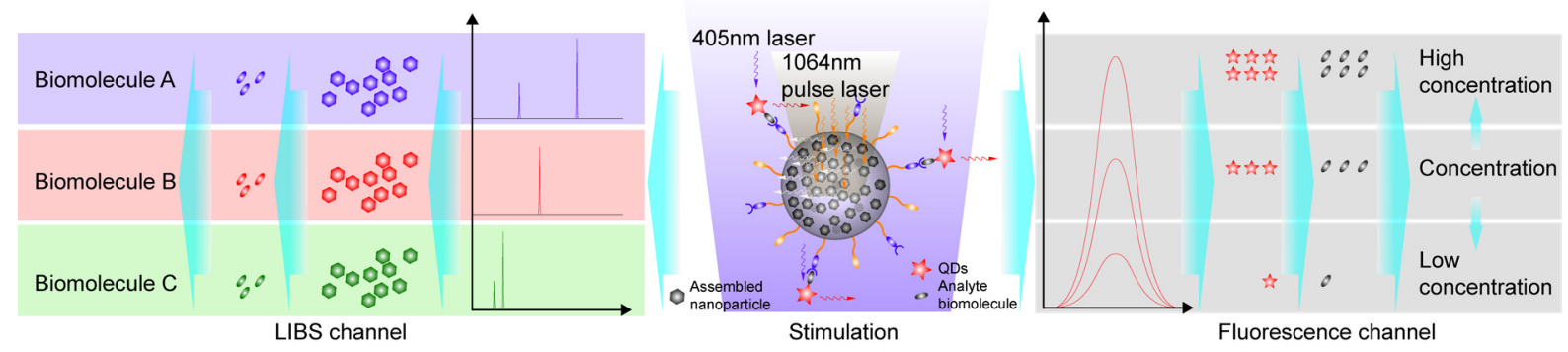

Fig. 1 The schematic of dual-channel design of optical demodulation system. 
real-time monitoring of fluorescence intensity with area $\mathrm{CCD}$ until maximum values were detected. Instantly, the fluorescence intensity was recorded. Afterward, the 405-nm laser was shutoff and the 1064-nm nanosecond pulse laser was turned on to induce samples. Meanwhile, the spectrograph-CCD system was triggered by $Q$ modulation signal output from the pulse laser and worked in trigger-acquisition mode to decode the induced microbead, finishing a single operation cycle. The acquisition cycle was kept running until the scanning of the slide was finished.

\subsection{Materials}

Monodispersed polystyrene microspheres $($ diameter $=30 \mu \mathrm{m}$, pore diameter $\approx 100 \mathrm{~nm}$ ) were purchased from the NanoMicro Research Center, Peking University (Beijing, China). Three types of QDs-labeled anti-IgG (525 nm@ goat antirabbit IgG, 565 nm@ goat antirat IgG, and 625 nm@ goat antimouse IgG) were obtained from Wuhan Jiayuan Quantum Dot Technological Development Corporation (Wuhan, China). Three types of IgG (rabbit, rat, and mouse) were purchased from Beyotime Biotechnology Co., Ltd. (Beijing, China). Ethanol, cyclohexane, hexadecane chloroform, 1-Ethyl-3-(3dimethylaminopropyl) carbodiimide (EDC), and N-hydroxysuccinimide (NHS) were obtained from Aladdin Industrial Corporation (Shanghai, China). Silver (Ag), magnesium oxide $(\mathrm{MgO})$, and zinc oxide $(\mathrm{ZnO})$ nanoparticles (NPs) were purchased from Macklin reagent Co., Ltd. (Shanghai, China). The selected plasma emission peaks as coding signals of nanoparticles are 520.9 and $546.5 \mathrm{~nm}$ for $\mathrm{Ag}, 516.7 \mathrm{~nm}$ for $\mathrm{MgO}$, and 472.2 and $481.0 \mathrm{~nm}$ for $\mathrm{ZnO}$.

\subsection{Preparation of Digital Encoded Suspension Array}

The high-temperature self-healing method ${ }^{27}$ was applied in the preparation of digitally encoded suspension array and the schematic is shown in Fig. 3. Concretely, $10 \mathrm{mg}$ of monodispersed polystyrene microspheres were dispersed into $4 \mathrm{~mL}$ of hexadecane and mixed by $5 \mathrm{~min}$ of ultrasonication. A hundredmicroliter nanoparticle solution $(20 \%, \mathrm{M} / \mathrm{M})$ was dispersed into $3 \mathrm{~mL}$ of chloroform and mixed for $5 \mathrm{~min}$ of ultrasonication too. Two dispersions were mixed together in a condenser pipe assembled flask and stirred with magnetism under $50^{\circ} \mathrm{C}$ for $1 \mathrm{~h}$; afterward, the dispersion was heated to $180^{\circ} \mathrm{C}$ and kept stirring until the reflux disappeared in the condenser pipe. Then the temperature was cooled to room temperature rapidly, and the reacted microspheres were filtrated by suction filtration and washed with ethanol and cyclohexane for several times to obtain digitally encoded microspheres. To create a suitable surface for bioprobe grafting, the prepared microspheres were dispersed into ethanol and dealt with an injected ozone air stream for $10 \mathrm{~min}$. Through oxidation, the surface of the microspheres was decorated with functional groups, such as hydroxyl and carboxyl. In the final step, the digitally encoded functional microspheres were mixed with bioprobes, which are IgGs in this experiment, for $2 \mathrm{~h}$ and accomplished probe grafting with the activation of EDC and NHS. To consume the remanent functional group on the surface, an excess $5 \%$ bull serum albumin solution [phosphate buffered saline (PBS), $10 \mathrm{mM}, \mathrm{pH}=7.4$ ] was used as a blocking buffer to mix with the microspheres for $36 \mathrm{~h}$ at $4^{\circ} \mathrm{C}$. The prepared suspension array was preserved in PBS for further utilization.

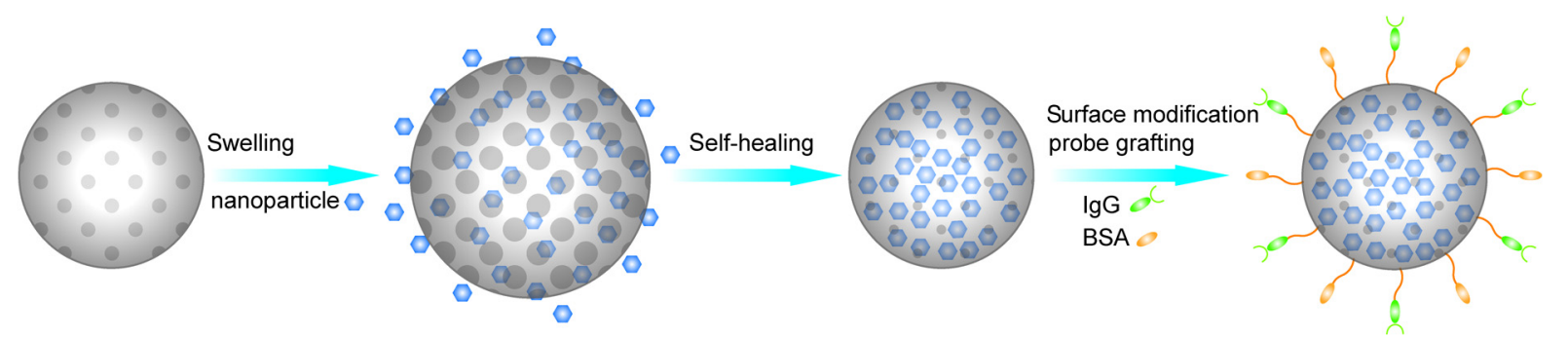

Fig. 3 The preparation of digitally encoded suspension array with high-temperature self-healing method.

(a)

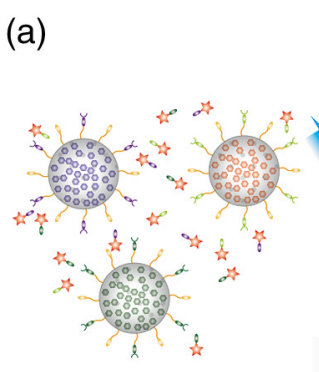

: QDs@Anti-lgG 1

QDs@Anti-IgG 2

\&QDs@Anti-IgG 3
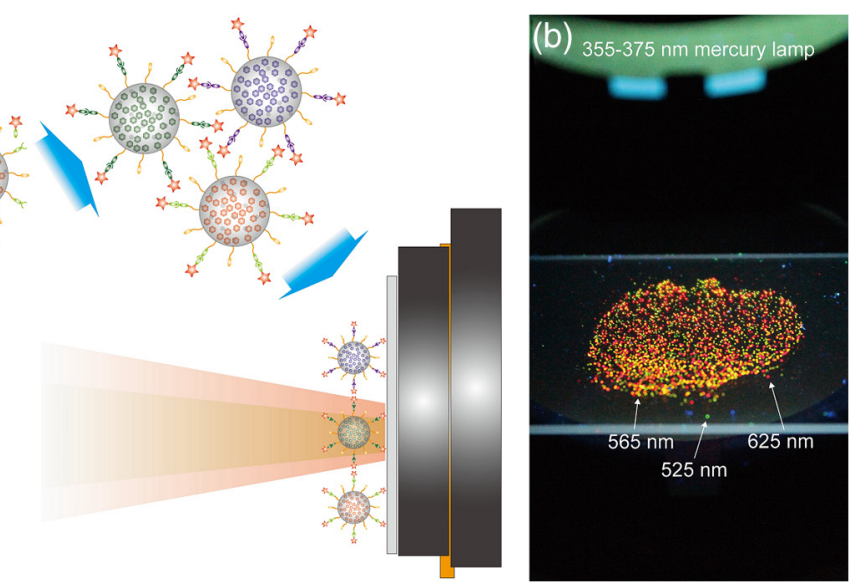

Fig. 4 (a) The detection mode of digitally encoded suspension array in fluoroimmunoassay. (b) The mercury lamp-stimulated images of reacted suspension array. 


\subsection{Multiplexed Analysis and Concentration Response of the System}

To verify the decoding performance of the optical demodulation system, we carried adsorption experiments to multiple biomolecules with specific fluorescent labels in the same sample. QDs with different colors were used to be the fluorescent labels of different types of anti-IgG in the experiments. It can be proved that the qualitative analysis could be successfully achieved by this system if the decoding result matches with the types of labels. We prepared three types of suspension arrays (SA1, SA2, and SA3) with AgNPs, MgNPs, and ZnONPs. The corresponding grafted bioprobes are rabbit IgG for SA1, rat IgG for SA2, and mouse $\mathrm{IgG}$ for SA3. Three types of suspension arrays were added into a mixed solution of $525 \mathrm{~nm} @$ goat antirabbit IgG, $565 \mathrm{~nm} @$ goat antirat IgG, and $625 \mathrm{~nm} @$ goat antimouse IgG and started another reaction for $30 \mathrm{~min}$ at $37^{\circ} \mathrm{C}$. The reacted microbeads were filtrated by suction filtration and placed on a glass slide for subsequent testing, as shown in Fig. 4. Theoretically, the detected fluorescence intensities would proportionally change in accordance with the variation in concentration of target molecules solution, providing the quantitative signal for analysis. To verify the concentration response of our system, we carried adsorption experiments to biomolecules with a series of concentration gradients. In detail, goat antirabbit IgG solutions with concentrations of $3.125 \times 10^{-10}, 6.25 \times 10^{-10}, 1.25 \times 10^{-9}, 2.5 \times 10^{-9}, 5 \times 10^{-9}$, and $1 \times 10^{-8} \mathrm{M}$ were tested as samples and the fluorescence intensities were recorded by the demodulation system.

\section{Results and Discussion}

\subsection{Laser-Induced Breakdown Spectrum of Encoded Materials and Digital Encoding Principle}

Stimulated by the nanosecond pulse laser, the high-energy plasma is formed on the surface of materials. ${ }^{28}$ The plasma emissions, including atomic lines, were radiated out with the procedure of plasma decay. In our measured spectrum range, from 450 to $760 \mathrm{~nm}$, the selected atomic lines match well with standard values, verifying the availability of the system in LIBS. As shown in Fig. 5, the 520.9 and $546.5 \mathrm{~nm}$ for $\mathrm{Ag}, 510.6,515.3$, and $521.8 \mathrm{~nm}$ for $\mathrm{Cu}_{2} \mathrm{O}, 516.7 \mathrm{~nm}$ for $\mathrm{MgO}$, and 472.2 and $481.0 \mathrm{~nm}$ for $\mathrm{ZnO}$ were selected as eight characteristic peaks for each element. To achieve the digital transformation of the laser-induced breakdown spectrum, we used 1 to represent the existence of the corresponding peak and 0 to represent that there is no peak in the encoding position. With this principle, these four types of encoding materials were labeled together with a binary sequence with eight characters. Further, to adequately utilize encoding materials, different nanoparticles could be assembled together in combination, thus greatly expanding the encoding count of suspension arrays.

\subsection{Preparation and Detection of Digital Encoded Suspension Array}

Assembling the selected nanoparticles into the carrier microspheres, we encoded suspension arrays with the above laserinduced breakdown spectrum and recognized microbeads with transformed digital encoding. In the process of assembling, the holes on the surface of monodispersed polystyrene microspheres were expanded with the effects of chloroform, the pore-foaming agent, and high temperature. Thus nanoparticles diffused into the holes under continuous stirring. While chloroform evaporated and the dispersion was cooled, the holes contracted rapidly and so locked nanoparticles inside. Once the microspheres were stimulated, the locked nanoparticles could produce plasma emissions and decode the suspension array with a specific laser-induced breakdown spectrum. After being encoded, the microspheres should experience surface modification and probe grafting to gain the ability of specifically detecting target molecules. Ozone possesses strong oxidizability so that it can break the carbon bond of organics to produce oxygen-containing groups, including hydroxyl and carboxyl. With this method, the surface of encoded monodispersed polystyrene microspheres turned out to be suitable for bioprobe grafting. In the experiment, QDs-labeled anti-IgG was used to play the role of the analyte. The IgG was grafted on the microspheres after surface modification to act as a bioprobe to capture the corresponding anti-IgG. This fluoroimmunoassay detection was carried in a solution of anti-IgG. To facilitate presentation,

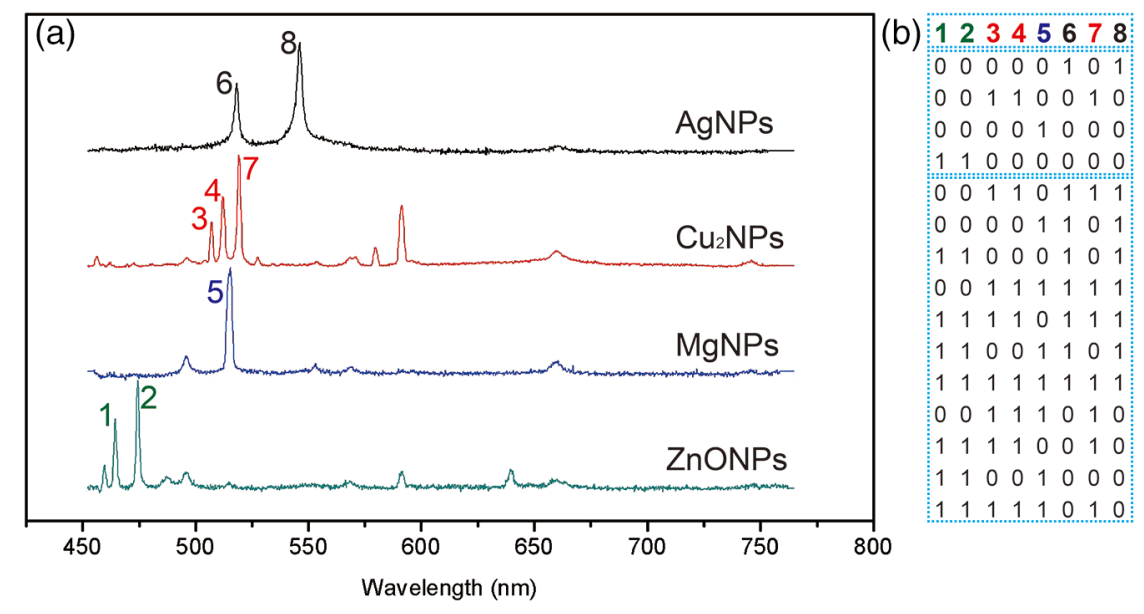

Fig. 5 (a) The laser-induced breakdown spectrum of encoding materials. (b) The digital transform principle of laser-induced breakdown spectrum, the binary sequences in upper grid are encoded by single type of elements and the binary sequences in under grid are encoded by different permutations and combinations of elements. 


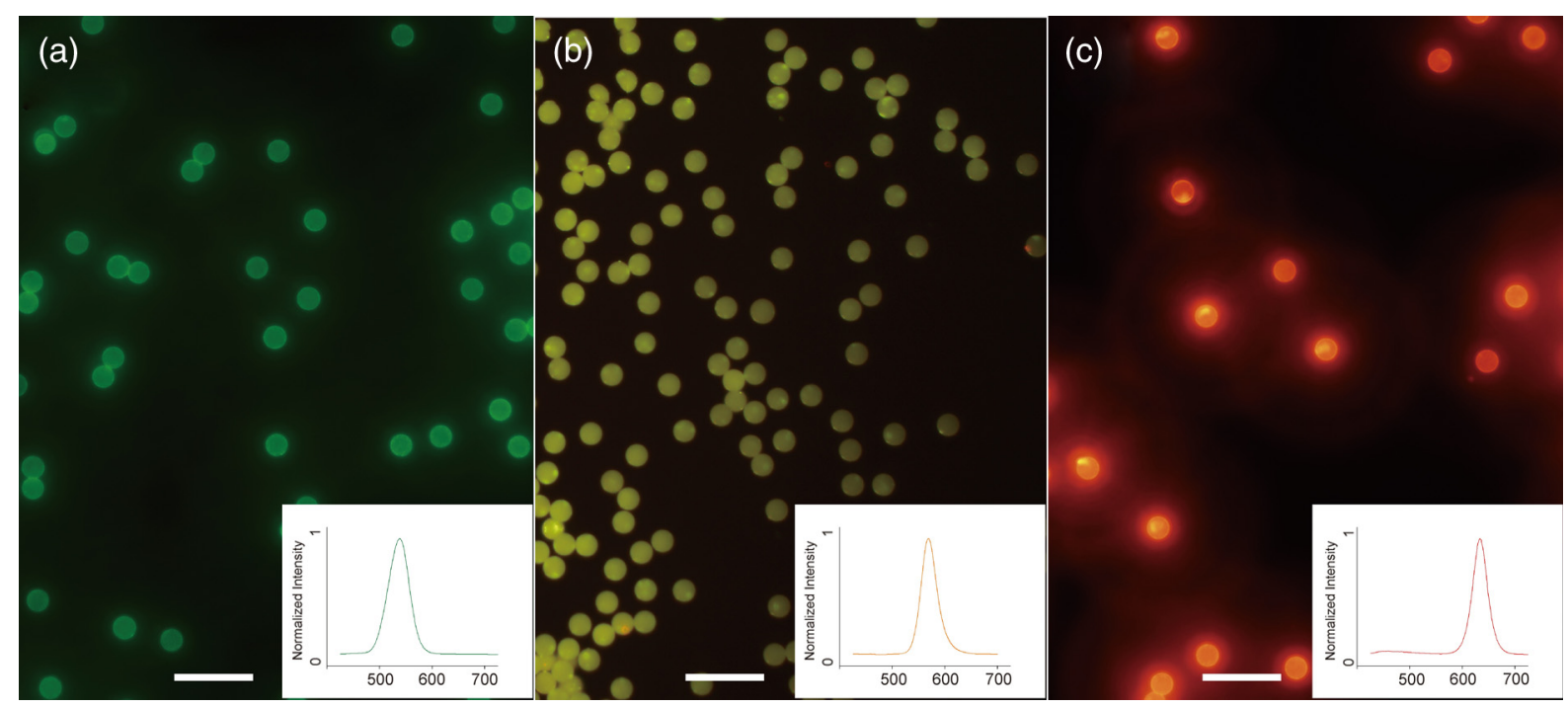

Fig. 6 The fluorescent microscope images of reacted (a) SA1, (b) SA2, and (c) SA3, the insets are the corresponding measured fluorescence spectrum. Scale bars are $100 \mu \mathrm{m}$.

the anti-IgG was labeled with QDs with different colors. The fluorescent microscope images of reacted suspension arrays are shown in Fig. 6 and the fluorescence spectrum were measured to be references of subsequent analysis.

\subsection{Multiplexed Analysis of the Optical Demodulation System}

The LIBS channel of the optical demodulation system can decode the digitally encoded suspension array with a laserinduced breakdown spectrum due to specifically assembled elements. And there are one-to-one correspondences between selected elements and grafted bioprobes in every type of suspension array. After antigen-antibody reaction in fluoroimmunoassay, the type of captured analyte can be recognized by decoding each microbead, the amount of analyte can be analyzed by counting the fluorescence intensity of labels. To demonstrate the decoding reliability of the optical demodulation system, we used QDs-labeled anti-IgG with different colors to be labeled analyte, the correspondences of bioprobe and analyte are listed as follows: rabbit IgG/525-nm QDs-labeled goat antirabbit IgG, rat IgG/565-nm QDs-labeled goat antirat IgG, and mouse IgG/625-nm QDs-labeled goat antimouse IgG. Three types of suspension arrays added into the mixture of three types of anti-IgG solutions and reacted for a while. The reacted microbeads were placed on a glass slide, the fluorescent microscope is shown in Fig. 7. The microbead experienced the 405-nm laser stimulation and nanosecond pulse laser induction successively. To reveal the relationship between LIBS spectra and corresponding types of biomolecules, we measured the fluorescence spectrum in the spectrograph-CCD system of the LIBS channel where the laser-induced breakdown spectra of microbeads were collected. As shown in Fig. 7, the decoding result matches well with the type of labeling antibodies, indicating the type of analyte. Hence, it is well proved that the optical demodulation system can achieve the multiplexed analysis of target biomolecules with a digitally encoded suspension array.

\subsection{Concentration Response of the Optical Demodulation System}

In the fluoroimmunoassay detection mode, the captured biomolecules were labeled by QDs, so the amount of captured biomolecules could be counted by collecting the fluorescence intensity of labels. In the fluorescence channel of the demodulation system, the 405-nm laser was used to stimulate the QDs label on

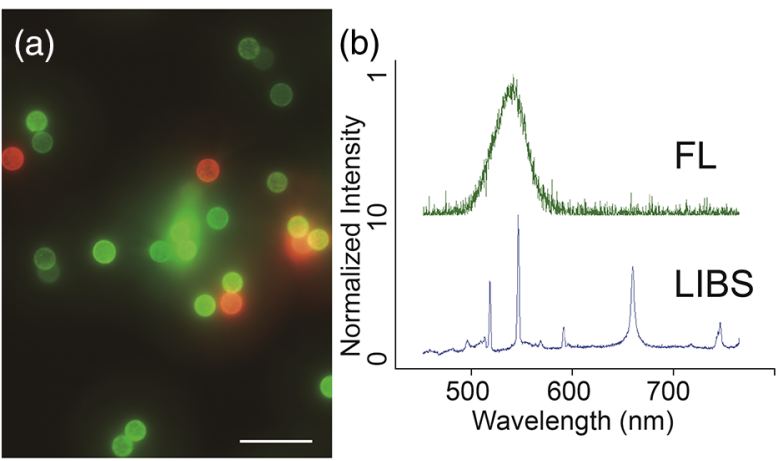

(c)

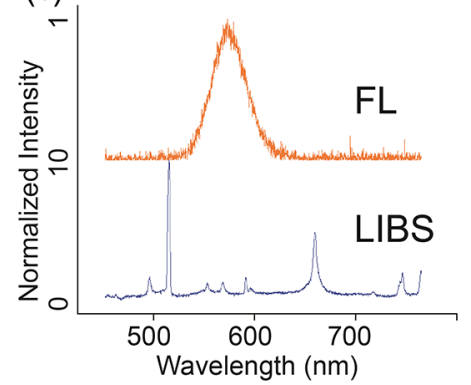

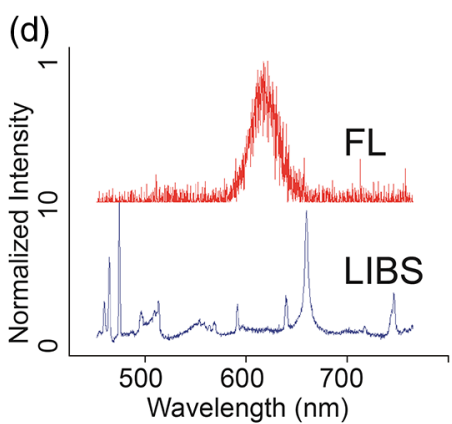

Fig. 7 (a) The fluorescence microscope image of mixed suspension array in multiplexed analysis. The fluorescence spectrum and laser-induced breakdown spectrum collected by the optical demodulation system: (b) SA1, (c) SA2, and (d) SA3. Scale bar is $100 \mu \mathrm{m}$. 


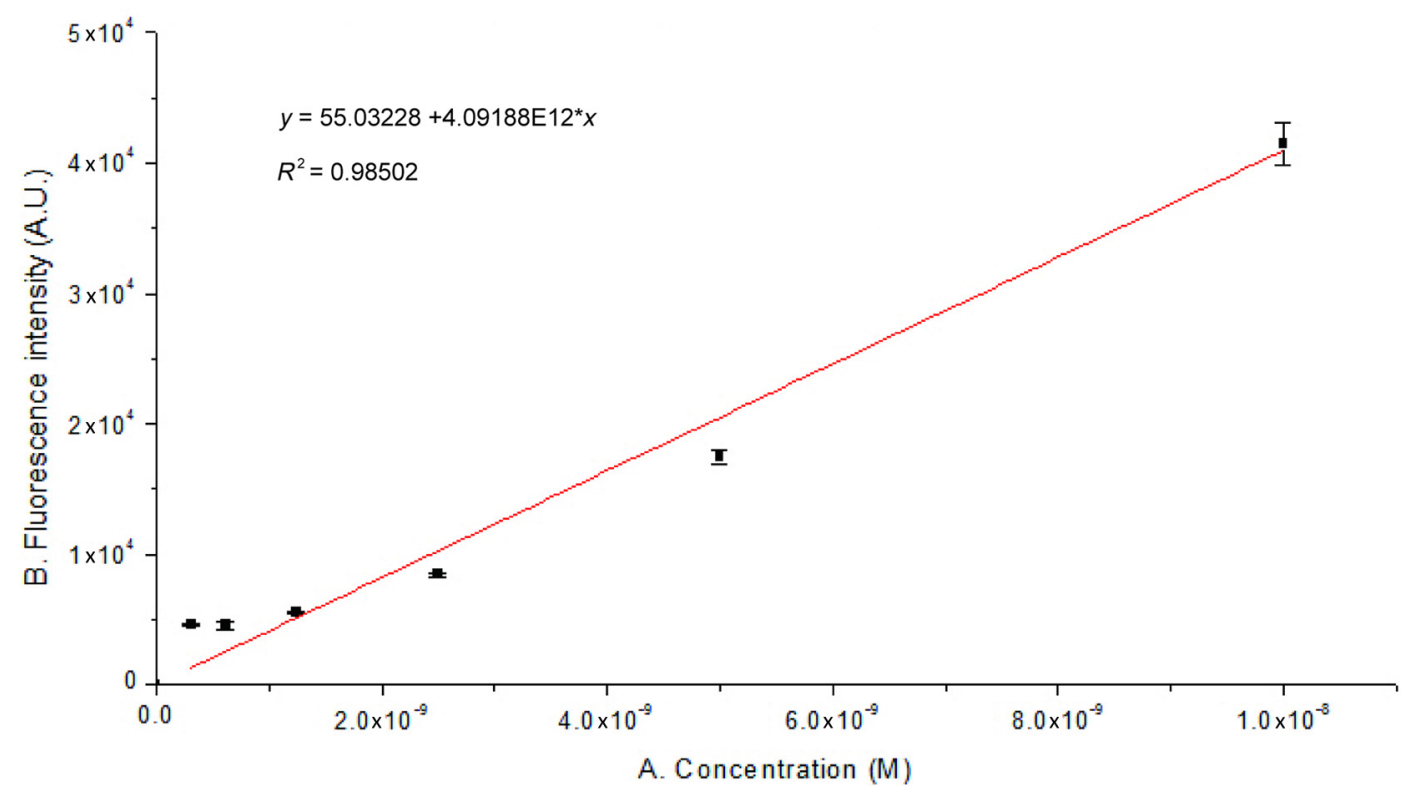

Fig. 8 The concentration response curve of fluorescence channel to SA1-based analysis to goat antirabbit lgG.

anti-IgG and the emission intensities were detected by coupled area $\mathrm{CCD}$, providing the quantitative information for biomolecule analysis. As an illustration, the gradient detection of anti- $\operatorname{IgG}$ was carried. Goat antirabbit $\operatorname{IgG}$ solutions with concentrations of $3.125 \times 10^{-10}, 6.25 \times 10^{-10}, 1.25 \times 10^{-9}$, $2.5 \times 10^{-9}, 5 \times 10^{-9}$, and $1 \times 10^{-8} \mathrm{M}$ were detected with SA1. The fluorescence of every type of reacted microbeads was measured by the demodulation system, and the intensities and corresponding concentrations were recorded and represented as a concentration curve, as shown in Fig. 8. Basically, the demodulation system has a linear response in our measurement scale, which verified the ability of the system to achieve quantitative analysis. The equation of concentration response curve is shown in the inset of Fig. 8. The empty sample was detected by digitally encoded suspension array and the optical demodulation system to measure the fluorescent system noise. The standard deviation of repeatedly measured system noise was calculated to be 220.2857654 (A.U.). According to the principle of triple standard difference method, the limit of detection of $1.48 \times 10^{-10} \mathrm{M}$ was achieved by the optical demodulation system, which is orders lower than traditional time-resolved fluoroimmunoassay and biochip systems encoded by fluorescence and the Raman spectrum. ${ }^{29-31}$

\section{Conclusion}

A dual-channel (fluorescence and LIBS)-coupled optical demodulation system for digitally encoded suspension arrays in fluoroimmunoassay was proved and its performance in multiplexed analysis of biomolecules was discussed. The nanoparticles assembled suspension array was decoded by stimulating and collecting the laser-induced breakdown spectrum in the LIBS channel. The stable and accurate encoding signal collected in the LIBS channel provided digital labels for the suspension array, guaranteeing the performance of qualitative recognition in multiplexed analysis. The captured target biomolecules on microbeads were labeled with QDs and the fluorescence intensity was measured in the fluorescence channel to accomplish quantitative analysis of targets. Therefore, various analytes could be analyzed in the same sample with our optical demodulation system, actually realizing the multiplexed analysis. Further, since the decoding and label detection were operated in LIBS and fluorescence channels, respectively, the cross talk between two types of signals was thoroughly avoided. This analysis mode not only kept the decoding procedure out of fluorescence interference, but also provided fluorescence detection a low-noise background, which is meaningful to improve the sensitivity of detection.

\section{Disclosures}

We declare that we have no financial and personal relationships with other people or organizations that can inappropriately influence our work, there is no professional or other personal interest of any nature or kind in any product, service and/or company that could be construed as influencing the position presented in, or the review of, the article entitled "Optical demodulation system for digitally encoded suspension array in fluoroimmunoassay."

\section{Acknowledgments}

This research was made possible with the financial support from National Science Foundation of China (NSFC) (61675113, 61527808, and 81571837); Science and Technology Research Program of Shenzhen City (JSGG20150331151536448, CXZZ20140416160720723, and JCYJ20160428182247170); 863 Project (2013AA032204).

\section{References}

1. J. M. Schwenk et al., "Antibody suspension bead arrays within serum proteomics," J. Proteome Res. 7(8), 3168-3179 (2008).

2. N. Zhang et al., "Luminescent solar concentrators with a bottommounted photovoltaic cell: performance optimization and power gain analysis," Chin. Opt. Lett. 15(6), 063501 (2017).

3. N. Liu et al., "Simultaneous detection for three kinds of veterinary drugs: chloramphenicol, clenbuterol and 17-beta-estradiol by highthroughput suspension array technology," Anal. Chim. Acta 632(1), 128-134 (2009). 
4. Y. Tanaka and S.-I. Wakida, "Time-shared optical tweezers with a microlens array for dynamic microbead arrays," Biomed. Opt. Express 6(10), 3670-3677 (2015).

5. B. Lendl et al., "Flow analysis-based surface-enhanced Raman spectroscopy employing exchangeable microbeads as SERS-active surfaces," Appl. Spectrosc. 54(7), 1012-1018 (2000).

6. R. Wilson, A. R. Cossins, and D. G. Spiller, "Encoded microcarriers for high-throughput multiplexed detection," Angew. Chem. Int. Ed. 45(37), 6104-6117 (2006).

7. B. Zhang et al., "A photonic crystal hydrogel suspension array for the capture of blood cells from whole blood," Nanoscale 8(6), 3841-3847 (2016).

8. N. T. Azimi et al., "Enzyme-linked immunoassays using nanosecond fluorometric detection," Appl. Spectrosc. 46(6), 994-998 (1992).

9. N. Ganesh et al., "Leaky-mode assisted fluorescence extraction: application to fluorescence enhancement biosensors," Opt. Express 16(26), 21626-21640 (2008).

10. M. Nichkova et al., "Multiplexed immunoassays for proteins using magnetic luminescent nanoparticles for internal calibration," Anal. Biochem. 369(1), 34-40 (2007).

11. Y. Zhao et al., "Quantum-dot-tagged bioresponsive hydrogel suspension array for multiplex label-free DNA detection," Adv. Funct. Mater. 20(6), 976-982 (2010)

12. Y. Long et al., "Multiplex immunodetection of tumor markers with a suspension array built upon core-shell structured functional fluorescence-encoded microspheres," Anal. Chim. Acta 665(1), 63-68 (2010).

13. Y. Leng et al., "Magnetic/fluorescent barcodes based on cadmium-free near-infrared-emitting quantum dots for multiplexed detection," $A d v$. Funct. Mater. 26(42), 7581-7589 (2016).

14. S. Chen et al., "Suspension array of ionic liquid or ionic liquid-quantum dots conjugates for the discrimination of proteins and bacteria," Anal. Chem. 87(21), 10902-10909 (2015).

15. X. Han et al., "Development and primary application of a fluorescent liquid bead array for the simultaneous identification of multiple genetically modified maize," Biosens. Bioelectron. 49, 360-366 (2013).

16. G.-X. Liang et al., "Near infrared sensing based on fluorescence resonance energy transfer between $\mathrm{Mn}$ :CdTe quantum dots and Au nanorods," Biosens. Bioelectron. 24(12), 3693-3697 (2009).

17. M. Cohen-Kashi, Y. Namer, and M. Deutsch, "Fluorescence resonance energy transfer imaging via fluorescence polarization measurement," J. Biomed. Opt. 11(3), 034015 (2006).
18. Y. Wang and L. V. Wang, "Förster resonance energy transfer photoacoustic microscopy," J. Biomed. Opt. 17(8), 086007 (2012).

19. N. L. Vasily et al., "Laser induced breakdown spectroscopy with picosecond pulse train," Laser Phys. Lett. 14(2), 026002 (2017).

20. C. Li et al., "Determinations of trace boron in superalloys and steels using laser-induced breakdown spectroscopy assisted with laserinduced fluorescence," Opt. Express 24(8), 7850-7857 (2016).

21. Y. Zhao et al., "Polarization-resolved laser-induced breakdown spectroscopy," Opt. Lett. 34(4), 494-496 (2009).

22. D. A. Rusak et al., "Investigation of the effect of target water content on a laser-induced plasma," Appl. Spectrosc. 51(11), 1628-1631 (1997).

23. F. Capitelli et al., "Determination of heavy metals in soils by laser induced breakdown spectroscopy," Geoderma 106(1-2), 45-62 (2002).

24. N. J. McMillan et al., "Geological applications of laser-induced breakdown spectroscopy," Geostand. Geoanal. Res. 38(3), 329-343 (2014).

25. S. Zhang et al., "Laser induced breakdown spectroscopy for local equivalence ratio measurement of kerosene/air mixture at elevated pressure," Opt. Lasers Eng. 50(6), 877-882 (2012).

26. A. C. Woods, C. G. Parigger, and J. O. Hornkohl, "Measurement and analysis of titanium monoxide spectra in laser-induced plasma," Opt. Lett. 37(24), 5139-5141 (2012).

27. Y. Liu et al., "Decoding of quantum dots encoded microbeads using a hyperspectral fluorescence imaging method," Anal. Chem. 87(10), 5286-5293 (2015).

28. W. Moćko et al., "Analysis of the plastic deformation of AISI 304 steel induced by the nanosecond laser pulse," Opt. Laser Technol. 90(7), 165-173 (2017).

29. G. J. Wang et al., "Time-resolved fluoroimmunoassay of plasma daidzein and genistein," Opt. Laser Technol. 65(6), 339-348 (2000).

30. Z. Chao et al., "Ultrasensitive time-resolved fluoroimmunoassay for saikosaponin a in Chaihu (Bupleuri Radix)," PLoS One 11(3), e0151032 (2016).

31. Y. Zhao et al., "Silver deposited polystyrene (PS) microspheres for surface-enhanced Raman spectroscopic-encoding and rapid label-free detection of melamine in milk powder," Talanta 113, 7-13 (2013).

Biographies for the authors are not available. 